\title{
OBSERVATIONS CONCERNING THE HARMFUL ENTOMOFAUNA FROM WINTER RAPESEED CROPS IN THE CONDITIONS OF CENTRAL OF MOLDAVA, BETWEEN YEARS 2014-2017
}

\author{
PAULA - LUCELIA URSACHE ${ }^{{ }^{*}}$, ELENA TROTUS ${ }^{1}$, \\ ALEXANDRA - ANDREEA BUBURUZ ${ }^{1}$ \\ ${ }^{1}$ Agricultural Research - Development Station Secuieni, str. Principală 371. Neamţ. \\ 617415, Romania
}

\begin{abstract}
During 2014-2017, 22 species were identified in the yellow bowl trap type installed in winter rapeseed crops established at A.R.S.D. Secuieni. Of these, 11 species are specific to winter rapeseed crops and 11 species were accidental identified because of the proximity with different agricultural crops (maize. sunflower. grain cereals). The highest abundance was registered by Epicometis hirta Poda species which accounted 914 specimens collected during the analyzed period. The species were integrated into classes of dominance ((D1 - subrecedent species. D2 - recedent species. D3 - subdominant species. D4 - dominant species and D5 - eudominant species). Meligethes aeneus F., Ceuthorrhyncus napi Gyll. and Epicometis hirta Poda. being considered eudominant species belonging to the D5 class. The Ceuthorrhynchus napi Gyll., Meligethes aeneus F., Epicomites hirta Poda., Psylliodes chrysocephala L., Subcoccinella 24 punctata L., Oulema melanopa L. species were classified in the constancy class - C4 - euconstant species. Meligethes aeneus F., Epicometis hirta Poda and Ceuthorrhynchus napi Gyll. species recorded the highest values for the index of ecological significance (W \%) and were classified in the W5 class - characteristic species. The collected entomofauna belongs to five orders: Coleoptera, Heteroptera, Lepidoptera,. Diptera and Hymenoptera. The highest number of species (17 species) and the highest number of collected speciemens (3246 specimens) belonged to the Coleoptera order.
\end{abstract}

Keywords: winter rapeseed crops, harmful entomofauna, ecological indicators, yellow bowl trap type

\section{INTRODUCTION}

Rapeseed crops involves a careful plant surveillance because it is one of the most ssusceptible crop to pest attack throught the phenological development of plants. Knowing the harmful species, which by attacking the different plant organs (leaves, stem, floral buds, flowers) leads to low productions of poor quality, it is important because teoretical knowledge combined with practical knowledge ensure the establishment of the suitable treatment moment [1-7].

The researches conducted to identify the pest atack correlated with influence of zonal agroecological factors has led to the establishment of the climate changes impact on winter rapeseed crops entomofauna. The drought which appears more frequently in the spring and summer months, the succession of high temperatures and low

\footnotetext{
* Corresponding author, email: p.ursache03@gmail.com

(C) 2017 Alma Mater Publishing House
} 
rainfall periods, especially in the winter months, determine the insect hibernation period to shorten causing great problems through the aggressive attacks and high densities of the key species.

In this paper we present data regarding the entomofauna collected from rapeseed crops in the conditions of Central of Moldova.

\section{EXPERIMENTAL SETUP}

The researches were conducted at the Agricultural Research - Development Station Secuieni - Neamt on the rapeseed crops. during 2014-2017 and consisted on collecting the harmful entomofauna from winter rapeseed crops, determining it and establishing of some ecological parameters represented by abundance, species dominance, constancy and index of ecological significance.

In spring, were installed yellow bowl trap type in the winter rapeseed crops, aiming to collect the harmful organisms, between the stem elongation phenophase until the end of seed ripening phenophase. The biological material was colected in Petri dishes, clear of vegetable remanins and analyzed in the laboratory at microscope, then grouped on species and orders.

The collected material was subjected to a mathematical analysis, obtaining a number of ecological parameters: abundance (A), dominance (D), constancy (C) and index of ecological significance (W), wich highlight the characteristics of the analyzed biocenoses.

The abundance (A \%) represents the total number of individuals of a species in the catch from a centain place on a given date. Based on the value of this indicator, the other indicators are calculated.

The dominance (D \%) shows the participation percentage of each species in the catch. Explains the relationship of a species herd with the sum of the individuals of the other associated species. This indicator is calculated according to the equation (1):

$$
D_{A}=\frac{N_{A} \cdot 100}{N_{1}}
$$

where:

$\mathrm{D}$ is dominance;

A - species abundance;

$\mathrm{N}_{\mathrm{A}}$ - the total number of individuals of A species;

$\mathrm{N}_{1}$ - the total number of individuals of the collected species.

Dominance classes include species whose spread percentage falls within the following values:

- D1-subrecedent species $\mathrm{P}<1.0 \%$;

- D2-recedent species $\mathrm{P}=1.1-2.0 \%$;

- D3-subdominant species $\mathrm{P}=2.1-5.0 \%$;

- D4-dominant species $\mathrm{P}=5.1-10.0 \%$;

- D5-eudominant species $\mathrm{P}>10.1 \%$.

The constancy (C \%) expresses the continuity of a species occurrence in the analyzed biotope. This characteristic is a structural indicator because it shows the participation proportion of a species in the biocenosis structure. The higher the value of the indicator, the more the species is better adapted to the conditions offered by the biotope.

The constancy is calculated according to the equation (2):

$$
C_{A}=\frac{n_{p} \cdot A}{N_{p}} \cdot 100
$$

where:

$\mathrm{C}_{\mathrm{A}}$ represent the constancy of A species; 
$\mathrm{n}_{\mathrm{p}} \cdot \mathrm{A}$ - the number of samples in which the A species occures;

$\mathrm{Np}$ - total number of collected samples.

Depending on the value of this indicator, the species are classified as follows:

- C1- accidental species $(1-25 \%)$;

- C2- accessories species(25.1-50\%);

- C3- constant species $(50.1-75 \%)$;

- C4- euconstant species(75.1-100\%).

The index of ecological significance (W \%) represents the relationship between the structural indicator $(C)$ and the productive indicator (D) and is calculated using equation (3):

$$
W_{A}=\frac{C_{A} \cdot D_{A} \cdot 100}{10000}
$$

where:

$\mathrm{W}_{\mathrm{A}}$ are the ecological significance index of A species;

$\mathrm{C}_{\mathrm{A}}$ - constancy of A species;

$\mathrm{D}_{\mathrm{A}}$ - dominance of A species.

Depending on the values obtained. the species are divided into the following classes:

$\mathrm{W} 1$ - accidental species $(\mathrm{W}<0.1 \%)$

$\mathrm{W} 2$ - accessories species $(\mathrm{W}=0.1-1.0 \%)$

W3- accessories species ( $\mathrm{W}=1.1 \%-5.1 \%)$

W4- characteristic species ( $\mathrm{W}=5.1 \%-10 \%)$

W5- characteristic species $(\mathrm{W}>10.1 \%$ )

\section{RESULTS AND DISCUSSION}

From the observations and determinations carried out on the entomofauna collected from winter rapeseed crops, it was found that it was made up of 22 species that totalized over the entire observation period (2014-2017) 3447 specimens (Table 1).

In 2016 were registered 1502 specimens and it was the year with the highest number of specimens collected, in 2015 were collected 1038 specimens, followed by the year 2014 with 618 specimens, and the lowest number of insects collected was recorded in 2017 - 289 specimens.

During the analyzed period, it was found that the indentified species had values ranging from one specimen at Agriotes spp. species to 914 specimens at Epicomites hirta Poda species. The highest number of collected specimens was recorded in the following species Meligethes aeneus F. (621 sp), species of Phyllotreta (494 sp). Ceuthorrhynchus napi Gyll. (406 sp), Ceuthorrhynchus assimillis Payk.(270 sp), Psylliodes chrysocephala L. (179 sp), Baris chlorizans Germ. (142 sp) and Athalia rosea L.(52 sp) (Table 1).

There were found other species of insects, which have been categorize as accidental species coming from proximity crops (maize. sunflower. grain cereals): Ostrinia nubilalis Hbn., Chlorops pumilionis Bjerk., Tanymecus dilaticollis Gyll., Crepidodera ferruginea Scopoli., Oscinella frit L., Oulema melanopa L., Eurygaster spp., but also entomophagus species (Subcoccinella 24 punctata L.).

Table 1. The colected entomofauna from the winter rapeseed crops at A.R.D.S. Secuieni during 2014-2017.

\begin{tabular}{|c|c|c|c|c|c|c|c|}
\hline \multirow[t]{2}{*}{ No. } & \multirow[t]{2}{*}{ Species } & \multirow[t]{2}{*}{ Order } & \multicolumn{4}{|c|}{ Collected entomofauna } & \multirow{2}{*}{$\begin{array}{c}\text { Total } \\
\text { specimens } \\
2014-2017\end{array}$} \\
\hline & & & 2014 & 2015 & 2016 & 2017 & \\
\hline 1 & Meligethes aeneus $\mathrm{F}$. & Coleoptera & 204 & 272 & 122 & 23 & 621 \\
\hline 2 & Phyllotreta atra Goeze & Coleoptera & 37 & 158 & 95 & 0 & 290 \\
\hline 3 & Phyllotetra nemorum L & Coleoptera & 12 & 142 & 29 & 0 & 183 \\
\hline 4 & Phyllotreta nigripes Fabr. & Coleoptera & 1 & 0 & 20 & 0 & 21 \\
\hline
\end{tabular}




\begin{tabular}{|c|c|c|c|c|c|c|c|}
\hline 5 & Psylliodes chrysocephala $\mathrm{L}$. & Coleoptera & 2 & 140 & 35 & 2 & 179 \\
\hline 6 & Baris chlorizans Germ. & Coleoptera & 12 & 71 & 59 & 0 & 142 \\
\hline 7 & Ceuthorrhynchus napi Gyll. & Coleoptera & 10 & 99 & 269 & 28 & 406 \\
\hline 8 & Ceuthorrhynchus assimillis Payk. & Coleoptera & 122 & 0 & 135 & 13 & 270 \\
\hline 9 & Ceuthorrhynchus pleurostigma Marsh. & Coleoptera & 1 & 0 & 3 & 0 & 4 \\
\hline 10 & Epicomites hirta Poda & Coleoptera & 81 & 75 & 573 & 185 & 914 \\
\hline 11 & Oulema melanopa $\mathrm{L}$. & Coleoptera & 2 & 3 & 11 & 16 & 32 \\
\hline 12 & Athalia rosea $\mathrm{L}$. & Hymenoptera & 0 & 33 & 15 & 4 & 52 \\
\hline 13 & Lygus pratensis $\mathrm{L}$. & Coleoptera & 3 & 1 & 54 & 0 & 58 \\
\hline 14 & Oscinella frit $\mathrm{L}$. & Diptera & 3 & 0 & 0 & 0 & 3 \\
\hline 15 & Tanymecus dilaticollis Gyll. & Coleoptera & 0 & 0 & 4 & 0 & 4 \\
\hline 16 & Crepidodera ferruginea Scopoli & Coleoptera & 5 & 0 & 0 & 0 & 5 \\
\hline 17 & Harpalus spp. & Coleoptera & 3 & 28 & 23 & 0 & 54 \\
\hline 18 & Subcoccinella 24 punctata $\mathrm{L}$. & Coleoptera & 21 & 5 & 26 & 10 & 62 \\
\hline 19 & Eurygaster $s p p$ & Heteroptera & 0 & 0 & 29 & 8 & 37 \\
\hline 20 & Agriotes spp. & Coleoptera & 0 & 1 & 0 & 0 & 1 \\
\hline 21 & Ostrinia nubilalis $\mathrm{Hbn}$. & Lepidoptera & 0 & 10 & 0 & 0 & 10 \\
\hline 22 & Chlorops pumilionis Bjerk.. & Diptera & 99 & 0 & 0 & 0 & 99 \\
\hline \multicolumn{3}{|c|}{ Total } & 618 & 1038 & 1502 & 289 & 3447 \\
\hline
\end{tabular}

From Table 2, it can be noted that the collected species were classified in dominance classes as follows:

- nine species belong to D1 class - subrecedent species with a spreading percentage below $1.0 \%$;

- three species were classified to D2 class - recedent species with a spreading rate ranging from $1.2 \%-2.0 \%$;

- two species were classified in D3 class - subdominant species whose spreading rates were between $2.1 \%$ $5.0 \%$;

- four species belong to D4 class - dominant species with spreading rates between $5.1 \%$ - $10 \%$;

- three species were classified in D5 class - eudominant species whose spread percentage was higher than $10.1 \%$.

Table 2. The classification on dominance classes of the species collected from the rapeseed crops at A.R.D.S. Secuieni.

\begin{tabular}{|c|c|c|c|c|c|}
\hline No. & $\begin{array}{l}\text { D1 - subrecedent } \\
\text { species }<1.1 \%\end{array}$ & $\begin{array}{l}\mathrm{D} 2-\text { recedent } \\
\text { species } \\
1.2 \%-2.0 \%\end{array}$ & $\begin{array}{l}\text { D3 - subdominant } \\
2.1 \%-5.0 \%\end{array}$ & $\begin{array}{c}\mathrm{D} 4-\text { dominant } \\
\text { species } \\
5.1 \%-10 \%\end{array}$ & $\begin{array}{l}\text { D5 - eudominant } \\
\text { species }>10.1 \%\end{array}$ \\
\hline 1 & Agriotes spp. & $\begin{array}{c}\text { Athalia rosea } \\
\text { L. } \\
\end{array}$ & $\begin{array}{l}\text { Baris chlorizans } \\
\text { Germ. }\end{array}$ & $\begin{array}{l}\text { Phyllotetra } \\
\text { nemorum L } \\
\end{array}$ & $\begin{array}{c}\text { Ceuthorrhynchus } \\
\text { napi Gyll. }\end{array}$ \\
\hline 2 & Oscinella frit L. & Harpalus spp. & $\begin{array}{c}\text { Chlorops } \\
\text { pumilionis Bjerk }\end{array}$ & $\begin{array}{c}\text { Psylliodes } \\
\text { chrysocephala } \mathrm{L} .\end{array}$ & $\begin{array}{l}\text { Meligethes } \\
\text { aeneus } \mathrm{F} \text {. }\end{array}$ \\
\hline 3 & $\begin{array}{c}\text { Tanymecus } \\
\text { dilaticollis Gyll. }\end{array}$ & $\begin{array}{c}\text { Lygus pratensis } \\
\text { L. }\end{array}$ & & $\begin{array}{l}\text { Ceuthorrhynchus } \\
\text { assimillis Payk }\end{array}$ & $\begin{array}{l}\text { Epicomites hirta } \\
\text { Poda } \\
\end{array}$ \\
\hline 4 & $\begin{array}{c}\text { Crepidodera } \\
\text { ferruginea Scopoli }\end{array}$ & & & $\begin{array}{l}\text { Phyllotreta atra } \\
\text { Goeze }\end{array}$ & \\
\hline 5 & $\begin{array}{c}\text { Ceuthorrhynchus } \\
\text { pleurostigma Marsh }\end{array}$ & & & & \\
\hline 6 & $\begin{array}{c}\text { Ostrinia nubilalis } \\
\text { Hbn }\end{array}$ & & & & \\
\hline 7 & $\begin{array}{c}\text { Phyllotreta nigripes } \\
\text { Fabr. }\end{array}$ & & & & \\
\hline 8 & Eurygaster spp & & & & \\
\hline 9 & Oulema melanopa L. & & & & \\
\hline Total & 9 species & 3 species & 2 species & 4 species & 3 species \\
\hline
\end{tabular}

Calculating the precentage of harmful entomofauna on dominance classes it was found that in D1 class, species with sporadic spreading were recorded $3.46 \%$ of the collected species. From D2 class, species with low spreading, belong $4.84 \%$ from the identified species. In D3 class, species with middle spread, were recorded $7.12 \%$ of the total, $27.34 \%$ belong to D4 class, dominant species, and in D5 class, eudominant species, were recorded $57.34 \%$ from the collected species (Figure 1). 


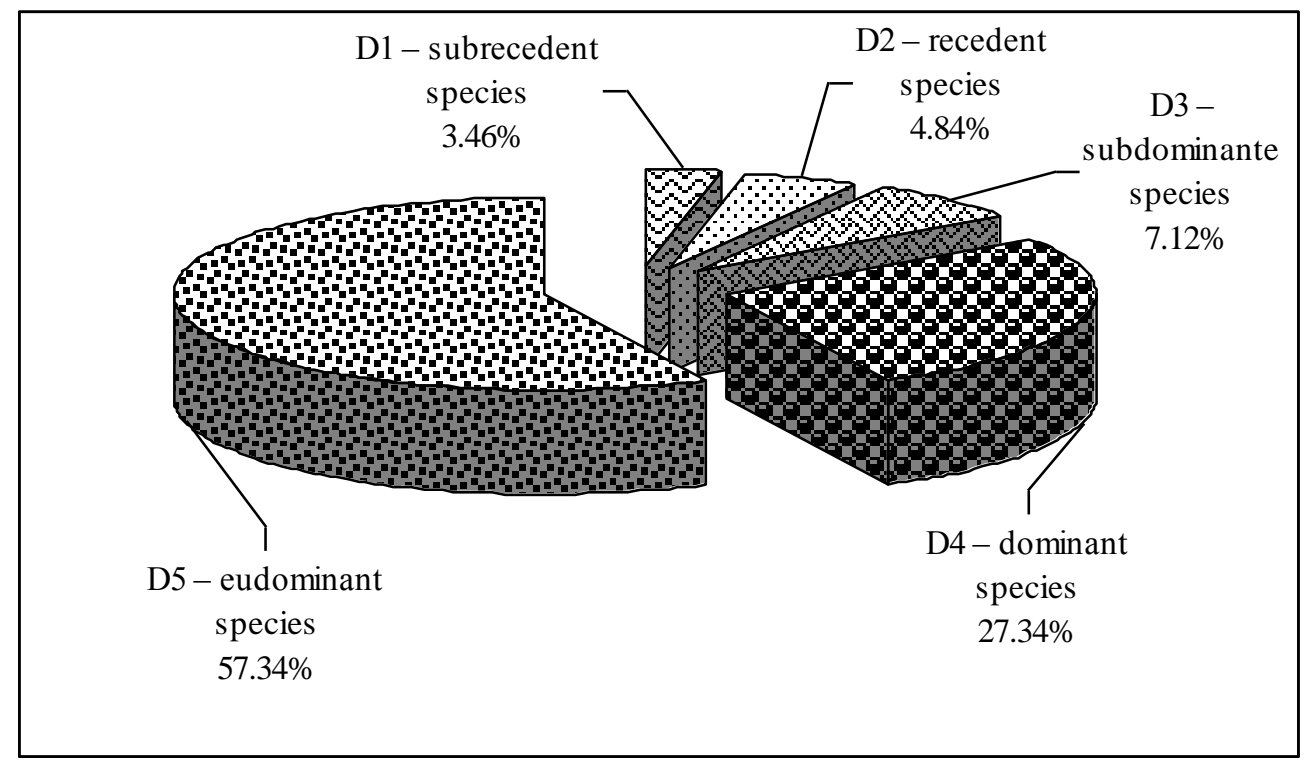

Fig. 1. The share of harmful species collected on dominance classes.

The species were distributed by constancy classes according to the obtained values (Table 3):

- six species had the constancy values between 1 and $25 \%$ belonging to $\mathbf{C 1}$ class - accidental species (Crepidodera ferruginea Scopoli., Tanymecus dilaticollis Gyll., Agriotes spp., Ostrinia nubilalis Hbn., Chlorops pumilionis Bjerk., Oscinella frit L);

- in C2 class - accessories species (25\% and $50 \%$ ). there were three species (Phyllotreta nigripes Fabr., Ceuthorrhynchus pleurostigma Marsh.,. Eurygaster spp.);

- to C3 class - constant species (50.1\%-75\%). belong seven species (Ceuthorrhynchus assimillis Payk., Phyllotreta atra Goeze, Phyllotetra nemorum L., Baris chlorizans Germ., Athalia rosea L.,. Lygus pratensis L., Harpalus spp);

- six species belong to C4 class - euconstant species (75.1\%-100 \%) (Ceuthorrhynchus napi Gyll., Meligethes aeneus F., Epicomites hirta Poda, Psylliodes chrysocephala L., Subcoccinella 24 punctata L., Oulema melanopa L.).

Table 3. The classification of the species collected in the yellow bowl trap type on constancy classes.

\begin{tabular}{|c|c|c|c|c|}
\hline \multirow[b]{2}{*}{ No. } & \multicolumn{4}{|c|}{ Constancy classes } \\
\hline & $\begin{array}{c}\mathrm{C}_{1}-\text { accidental species } \\
(1-25 \%)\end{array}$ & $\begin{array}{c}\mathrm{C}_{2}-\text { accessories } \\
\text { species }(25.1-50 \%)\end{array}$ & $\begin{array}{c}\mathrm{C}_{3}-\text { constant species } \\
(50.1-75 \%)\end{array}$ & $\begin{array}{c}\mathrm{C}_{4}-\text { euconstant species } \\
(75.1-100 \%)\end{array}$ \\
\hline 1 & Agriotes spp. & $\begin{array}{c}\text { Ceuthorrhynchus } \\
\text { pleurostigma } \text { Marsh }\end{array}$ & $\begin{array}{c}\text { Ceuthorrhynchus } \\
\text { assimillis Payk }\end{array}$ & $\begin{array}{l}\text { Ceuthorrhynchus napi } \\
\text { Gyll. }\end{array}$ \\
\hline 2 & Oscinella frit $\mathrm{L}$. & $\begin{array}{l}\text { Phyllotreta nigripes } \\
\text { Fabr. }\end{array}$ & Phyllotreta atra Goeze & Meligethes aeneus $\mathrm{F}$. \\
\hline 3 & $\begin{array}{c}\text { Tanymecus dilaticollis } \\
\text { Gyll. }\end{array}$ & Eurygaster spp & Baris chlorizans Germ. & Epicomites hirta Poda \\
\hline 4 & $\begin{array}{c}\text { Crepidodera ferruginea } \\
\text { Scopoli }\end{array}$ & & Phyllotetra nemorum $\mathrm{L}$ & $\begin{array}{c}\text { Psylliodes } \\
\text { chrysocephala } \mathrm{L} .\end{array}$ \\
\hline 5 & Ostrinia nubilalis $\mathrm{Hbn}$ & & Athalia rosea $\mathrm{L}$. & $\begin{array}{c}\text { Subcoccinella } 24 \\
\text { punctata } L .\end{array}$ \\
\hline 6 & $\begin{array}{c}\text { Chlorops pumilionis } \\
\text { Bjerk. }\end{array}$ & & Harpalus spp. & Oulema melanopa L. \\
\hline 7 & & & Lygus pratensis L. & \\
\hline Total & 6 species & 3 species & 7 species & 6 species \\
\hline
\end{tabular}


Depending on the index of ecological significance (W), the species were classified as follow (Table 4):

- six species (Agriotes spp..Oscinella frit L., Tanymecus dilaticollis Gyll., Crepidodera ferruginea Scopoli., Ostrinia nubilalis Hbn,. Ceuthorrhynchus pleurostigma Marsh.) are part of W1 class $(<0.1 \%)$;

- in W2 class (0.1-1.0\%) belong four species (Phyllotreta nigripes Fabr., Chlorops pumilionis Bjerk., Eurygaster spp., Oulema melanopa L.);

- six species ( Athalia rosea L., Harpalus spp., Lygus pratensis L., Subcoccinella 24 punctata L., Baris chlorizans Germ., Phyllotetra nemorum L.) belong to W3 class (1.1-5.0 \%);

- three species (Ceuthorrhynchus assimillis Payk., Psylliodes chrysocephala L., Phyllotreta atra Goeze) were recorded to $\mathbf{W} 4$ class $(5.1-10.0 \%)$;

- from W5 class $(>10.0 \%)$ are three species (Meligethes aeneus F., Epicomites hirta Poda, Ceuthorrhynchus napi Gyll.).

Table 4. The classification of the species collected in the yellow bowl trap type according to the index of ecological significance $(\mathrm{W})$ classes.

\begin{tabular}{|c|c|c|c|c|c|}
\hline \multirow[t]{2}{*}{ No. } & \multicolumn{5}{|c|}{ Index of ecological significance $(\mathrm{W})$ classes } \\
\hline & $\mathrm{W}_{1}<0.1 \%$ & $\mathrm{~W}_{2}-0.1-1.0 \%$ & $\mathrm{~W}_{3}-1.1-5.0 \%$ & $\mathrm{~W}_{4}-\mathbf{5 . 1 - 1 0 . 0 \%}$ & $\mathrm{W}_{5}->10.0 \%$ \\
\hline 1 & Agriotes spp. & $\begin{array}{c}\text { Phyllotreta nigripes } \\
\text { Fabr. }\end{array}$ & $\begin{array}{l}\text { Athalia rosea } \\
\text { L. }\end{array}$ & $\begin{array}{c}\text { Psylliodes } \\
\text { chrysocephala } \\
\text { L. }\end{array}$ & $\begin{array}{l}\text { Ceuthorrhynch } \\
\text { us napi Gyll. }\end{array}$ \\
\hline 2 & Oscinella frit $\mathrm{L}$. & Eurygaster spp & Harpalus spp. & $\begin{array}{l}\text { Ceuthorrhynchu } \\
\text { s assimillis Payk }\end{array}$ & $\begin{array}{l}\text { Meligethes } \\
\text { aeneus } \mathrm{F} .\end{array}$ \\
\hline 3 & $\begin{array}{c}\text { Tanymecus } \\
\text { dilaticollis } \text { Gyll. }\end{array}$ & $\begin{array}{c}\text { Chlorops pumilionis } \\
\text { Bjerk. }\end{array}$ & $\begin{array}{c}\text { Lygus pratensis } \\
\text { L. }\end{array}$ & $\begin{array}{c}\text { Phyllotreta atra } \\
\text { Goeze }\end{array}$ & $\begin{array}{l}\text { Epicomites } \\
\text { hirta } \text { Poda }\end{array}$ \\
\hline 4 & $\begin{array}{c}\text { Crepidodera } \\
\text { ferruginea } \text { Scopoli }\end{array}$ & Oulema melanopa $\mathrm{L}$. & $\begin{array}{l}\text { Subcoccinella } \\
24 \text { punctata } L .\end{array}$ & & \\
\hline 5 & $\begin{array}{c}\text { Ceuthorrhynchus } \\
\text { pleurostigma } \\
\text { Marsh }\end{array}$ & & $\begin{array}{c}\text { Baris } \\
\text { chlorizans } \\
\text { Germ. }\end{array}$ & & \\
\hline 6 & $\begin{array}{l}\text { Ostrinia nubilalis } \\
\text { Hbn }\end{array}$ & & $\begin{array}{l}\text { Phyllotetra } \\
\text { nemorum } \mathrm{L}\end{array}$ & & \\
\hline Total & 6 specii & 4 specii & 6 specii & 3 specii & 3 specii \\
\hline
\end{tabular}

Grouping the collected species on systematic orders showed that the analyzed entomofauna classified in five orders: Coleoptera, Heteroptera, Lepidoptera, Diptera and Hymenoptera (Figure 2).

From Coleoptera order were registered most of the species spread in the rapeseed crops, respectively 17 species, to Diptera order belongs tow species and the Heteroptera, Lepidoptera and Hymenoptera orders were represented by one species each (table 5).

Table 5. The distribution of collected species from rapeseed crops on systematic orders.

\begin{tabular}{|c|c|c|c|c|c|}
\hline No. & Coleoptera & Heteroptera & Lepidoptera & Diptera & Hymenoptera \\
\hline 1 & Meligethes aeneus F. & Eurygaster spp & $\begin{array}{c}\text { Ostrinia } \\
\text { nubilalis Hbn. }\end{array}$ & Oscinella frit L. & $\begin{array}{c}\text { Athalia rosea } \\
\text { L. }\end{array}$ \\
\hline 2 & Phyllotreta atra Goeze & & & $\begin{array}{c}\text { Chlorops } \\
\text { pumilionis } \\
\text { Bjerk.. }\end{array}$ & \\
\hline 3 & Phyllotetra nemorum L. & & & & \\
\hline 4 & $\begin{array}{r}\text { Phyllotreta nigripes } \\
\text { Fabr. }\end{array}$ & & & & \\
\hline 5 & $\begin{array}{r}\text { Psylliodes chrysocephala } \\
\text { L. }\end{array}$ & & & & \\
\hline 6 & Baris chlorizans Germ. & & & & \\
\hline 7 & $\begin{array}{r}\text { Ceuthorrhynchus napi } \\
\text { Gyll. }\end{array}$ & & & & \\
\hline
\end{tabular}




\begin{tabular}{|c|c|l|l|l|l|}
\hline 8 & $\begin{array}{c}\text { Ceuthorrhynchus } \\
\text { assimillis Payk. }\end{array}$ & & & & \\
\hline 9 & $\begin{array}{c}\text { Ceuthorrhynchus } \\
\text { pleurostigma Marsh. }\end{array}$ & & & & \\
\hline 10 & Epicomites hirta Poda & & & & \\
\hline 11 & Oulema melanopa L. & & & & \\
\hline 12 & Lygus pratensis L. & & & & \\
\hline 13 & $\begin{array}{c}\text { Tanymecus dilaticollis } \\
\text { Gyll. }\end{array}$ & & & & \\
\hline 14 & $\begin{array}{c}\text { Crepidodera ferruginea } \\
\text { Scopoli }\end{array}$ & & & & \\
\hline 15 & Harpalus spp. & & & & \\
\hline 16 & $\begin{array}{c}\text { Subcoccinella } 24 \\
\text { punctata L. }\end{array}$ & & & & \\
\hline 17 & Agriotes spp. & & & & \\
\hline Total & 17 species & one species & one species & 2 species & one species \\
\hline
\end{tabular}

By calculating the percentage of orders after the number of species it was found that: Coleoptera order had the maximum share of $77.4 \%$, followed by Diptera order which had a share of $9.1 \%$ and then by Hymenoptera. Heteroptera and Lepidoptera orders with $4.5 \%$ each (Figure 2).

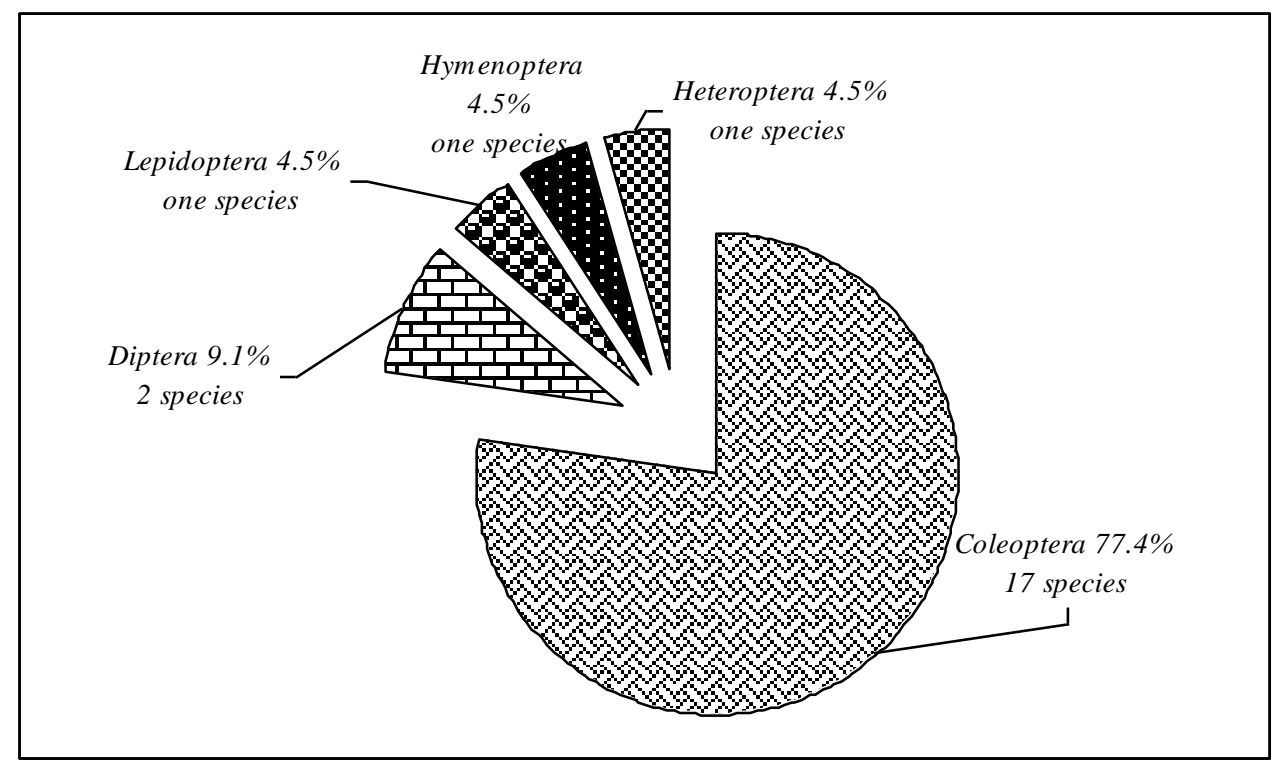

Fig. 2. The orders share depending on the number of species collected from rapeseed crops.

Within the Coleoptera order the highest share of $28.07 \%$ belongs to Epicomites hirta Poda species, followed by Meligethes aeneus F. with $19.02 \%$ and Ceuthorrynchus napi Gyll. with 12.4 7\%. The lowest shares were recorded by the species: Agriotes spp. with $0.03 \%$, Tanymecus dilaticollis Gyll with $0.12 \%$ and Crepidodera ferruginea Scopoli with $0.15 \%$ (Figure 3). 


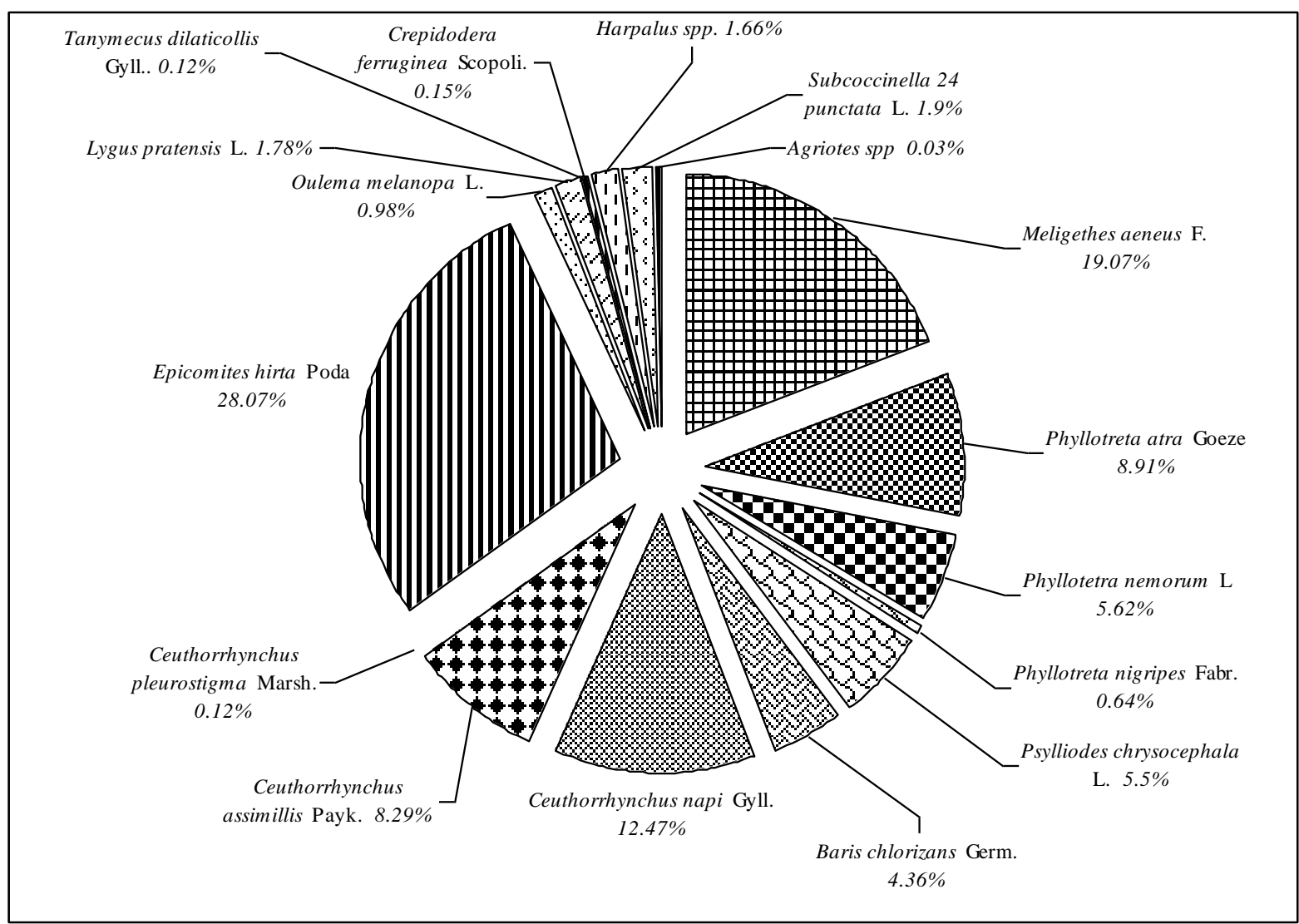

Fig. 3. The species share from Coleoptera order in rapeseed crops.

\section{CONCLUSIONS}

During 2014-2017 were identified 22 species using the yellow bowl trap type installed in winter rapeseed crops established at A.R.S.D. Secuieni.

Of these, 11 species are specific to winters rapeseed crops and 11 species were accidental identified due to their proximity to different agricultural crops (maize, sunflower, grain cereals).

The highest abundance was recorded by Epicometis hirta Poda species with 914 specimens collected during the analyzed period.

The species were classified into dominance classes ((D1 - subrecedent species. D2 - recedent species. D3 subdominant species. D4 - dominant species and D5 - eudominant species). Meligethes aeneus F., Ceuthorrhyncus napi Gyll. and Epicometis hirta Poda. being considered as eudominant species belonging to D5 class.

The Ceuthorrhynchus napi Gyll., Meligethes aeneus F., Epicomites hirta Poda, Psylliodes chrysocephala L., Subcoccinella 24 punctata L., Oulema melanopa L., species have been classified into C4 constancy class euconstant species.

Meligethes aeneus F., Epicometis hirta Poda and Ceuthorrhynchus napi Gyll. species recorded the highest values on the ecological significance index (W\%) and were classified in W5 class - characteristic species.

The analyzed entomofauna belongs to five orders: Coleoptera, Heteroptera, Lepidoptera, Diptera and Hymenoptera. The higest number of species (17 species) and the highest number of collected speciemens (3246 specimens) belonged to the Coleoptera order 


\section{REFERENCES}

[1] Buburuz, A.A.,Trotus, E.,Talmaciu, M., Results on specific harmful entomofauna from rapeseed crops in the Central Moldavian Plateau conditions, Agronomy series, vol 55, 2012, p. $305-308$.

[2] Buburuz, A.A., Trotus, E., Zaharia, P., The rape crop protection against harmful organisms, under specific conditions from Center of Moldavia, AN. I.N.C.D.A. Fundulea., vol. LXXX., 2012, p. 199-209.

[3] Gorenschi (Buburuz), A.A., Research in the useeful entomofauna from rapeseed crops, in A.R.D.S. SecuieniNeamt conditions, depending on the chemical treatment applied for pest control, Phd thesis, USAMV, Iasi, 2014.

[4] Trotus, E., Research on the knowledge and control of specific pests from rapeseed crops under the conditions of the Center of Moldova, Lucrarile simpozionului ştiinţific, USAMV Iasi, Romania, 2003.

[5] Trotus, E., Let us know in good time the danger posed by rapeseed pests to the production, InfoAmsem, no. 1,2003 , p. $4-6$.

[6] Trotus, E., Evolution of harmful entomofauna in rapeseed crops in the Center of Moldova, 45 years of scientific activity SCDA Secuieni - Anniversary, Ed. Ion Ionescu de la Brad, Iasi, 2007.

[7] Trotus, E., Popov, C., Rasnoveanu, L., Stoica, V., Naie, M., Mureşan, F., Management of rapeseed crops against insect pest attack, AN. I.N.C.D.A. Fundulea, vol. LXXVII, 2009, p. 211-222. 